\title{
Droplets impaling on a cone
}

\author{
Guillaume Durey $\odot,{ }^{1, *}$ Quentin Magdelaine $\odot,{ }^{1}$ Mathias Casiulis $\odot,{ }^{1}$ Hoon Kwon, ${ }^{1}$ Julien Mazet, ${ }^{1,2}$ \\ Pierre Chantelot, ${ }^{3,4, \dagger}$ Anaïs Gauthier, ${ }^{3,4}$ Christophe Clanet, ${ }^{3,4}$ and David Quéré ${ }^{3,4}$ \\ ${ }^{1}$ The Lutetium Project, ESPCI Paris, Université PSL, Paris, France \\ ${ }^{2}$ Conservatoire National Supérieur de Musique et de Danse de la Ville de Paris, Université PSL, Paris, France \\ ${ }^{3}$ PMMH, UMR CNRS 7636, ESPCI Paris, Université PSL, Sorbonne Université, \\ Université de Paris, Paris, France \\ ${ }^{4}$ LadHyX, UMR CNRS 7646, École Polytechnique, Palaiseau, France
}

(Received 21 August 2020; published 12 November 2020)

\begin{abstract}
This paper is associated with a video winner of a 2019 American Physical Society's Division of Fluid Dynamics (DFD) Milton van Dyke Award for work presented at the DFD Gallery of Fluid Motion. The original video is available online at the Gallery of Fluid Motion, https://doi.org/10.1103/APS.DFD.2019.GFM.V0013.
\end{abstract}

DOI: 10.1103/PhysRevFluids.5.110507

Superhydrophobic materials are known for two main properties: They evacuate water drops when they are tilted, and they reflect them upon impact [1]. Both properties are key to many applications where wetting, impregnation, or drop-induced optical distortion need to be prevented. Local modifications of the substrate geometry, on a scale intermediate between that of the microtexture needed for superhydrophobicity and that of the drop, can further improve the water or ice repellency of superhydrophobic materials [2,3]. Here, we consider a geometry for which we anticipate a competition between bouncing and sliding, namely that of a superhydrophobic cone on which we observe the impacts of water drops.

Figure 1 shows successive snapshots of the impact of a drop of distilled water with velocity $1 \mathrm{~m} / \mathrm{s}$ on a superhydrophobic cone with aperture $\alpha=100^{\circ}$, a situation where the two repellent functions of the solid somehow equilibrate. The liquid first spreads along the surface of the cone and forms a rim. Its motion has both a horizontal and a vertical component, which markedly differs from spreading on a flat surface, where the vertical motion of the falling drop becomes fully horizontal at the rim. At time $t=2.4 \mathrm{~ms}$, the tip of the cone punctures the liquid, which forms a thin film that dewets outwards until it collides with the outer rim. This collision leads to the formation of a liquid torus, whose behavior depends on the aperture of the cone: For small apertures $\left(<90^{\circ}\right)$, the liquid keeps running down the solid, while for flatter cones $\left(>100^{\circ}\right)$, the ring bounces up. A remarkable behavior arises at intermediate values of $\alpha$ : The vertical momenta of the two dewetting rims cancel out, generating a purely radial motion; the liquid torus remains (transiently) horizontal and is stretched. At $t=7.2 \mathrm{~ms}$, corrugations appear and grow until the ring fully breaks up into liquid pearls, a consequence of the Rayleigh-Plateau instability. This fragmentation contrasts with

\footnotetext{
*guillaume.durey@espci.org

†p.chantelot@gmail.com
}

Published by the American Physical Society under the terms of the Creative Commons Attribution 4.0 International license. Further distribution of this work must maintain attribution to the author(s) and the published article's title, journal citation, and DOI. 

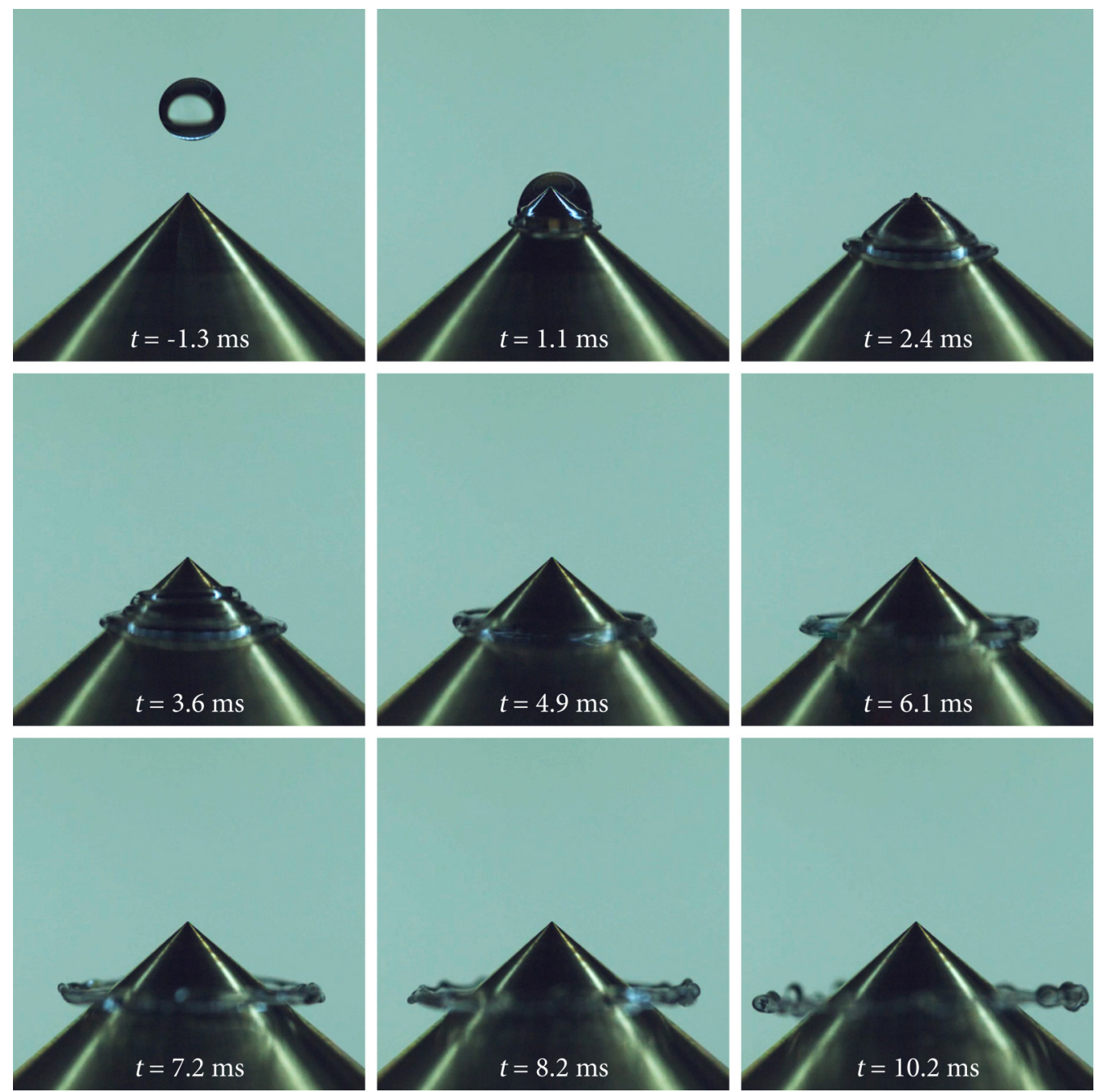

FIG. 1. Image sequence of a water drop impacting a superhydrophobic cone with aperture $100^{\circ}$. The liquid is repelled in the shape of a ring whose motion is confined in a horizontal plane. The ring expands and then breaks up into smaller liquid fragments through a Rayleigh-Plateau instability. https://doi.org/10.1103/APS. DFD.2019.GFM.V0013.

the rebound property at a similar impact velocity on flat superhydrophobic surfaces where a drop spreads, recoils, and finally takes off as a whole.

Hence, by altering the geometry of our substrates, we combine the sliding and bouncing properties of superhydrophobic materials, with a twist. We create rare fluid objects, tori, whose unusual dynamics prevent them from contracting into a single spherical drop, enforcing fragmentation of the liquid.

This research experiment was turned into an outreach video by the members of the Lutetium Project, a YouTube channel aimed at showcasing modern experimental science with an artistic spin [4]. The Paris-based team comprises young researchers in fluid dynamics and soft matter physics, graphic designers, music composers, and computer graphics artists. Other experiments set to music from the Lutetium Project include bouncing and walking droplets, the transition from laminar to turbulent flow, self-propelled microdroplets, topological defects waltzing in spherical shells of a 
liquid crystal [5], and the emulsification of water-alcohol mixtures on a layer of oil, a phenomenon known as Marangoni bursting [6,7].

The first shots of the video visually introduce a series of concepts from interfacial hydrodynamics to the general public, here using the remarkable bounces observed on superhydrophobic materials. The consequences of a modification of the substrate geometry are then showcased, building up to impacts on cones, and culminating with the situation discussed above where sliding and bouncing remarkably equilibrate.

Throughout the video, the physics is underscored by an original soundtrack-a defining feature of the Lutetium Project. While the melodies in the background evoke buoyancy, weightlessness, and the slow passing of time characteristic of high-speed imagery, the high-pitched notes in the foreground convey the bouncing, impalement, and fragmentation of the droplets after their interaction with the substrates. The music is characterized by a sustained crescendo, which builds up tension until the explosive final shot, featuring a crown splash.

The Lutetium Project acknowledges financial support from Espace des sciences Pierre-Gilles de Gennes, Université PSL (IDEX ANR-10-IDEX-0001-02 PSL), ESPCI Paris, ESPCI Alumni, and Fonds ESPCI Paris.

[1] R. Blossey, Self-cleaning surfaces - virtual realities, Nat. Mater. 2, 301 (2003).

[2] J. C. Bird, R. Dhiman, H.-M. Kwon, and K. K. Varanasi, Reducing the contact time of a bouncing drop, Nature (London) 503, 385 (2013).

[3] Y. Liu, L. Moevius, X. Xu, T. Qian, J. M. Yeomans, and Z. Wang, Pancake bouncing on superhydrophobic surfaces, Nat. Phys. 10, 515 (2014).

[4] https://youtube.com/thelutetiumproject.

[5] G. Durey, Q. Magdelaine, M. Kasiulis, H. Kwon, J. Mazet, A. Darmon, M. Benzaquen, O. Dauchot, and T. Lopez-Leon, Video: Topological microfluidics: Waltzing defects in double emulsions of a chiral liquid crystal, https://doi.org/10.1103/APS.DFD.2019.GFM.V0016.

[6] G. Durey, H. Kwon, Q. Magdelaine, M. Casiulis, J. Mazet, L. Keiser, H. Bense, J. Bico, P. Colinet, and E. Reyssat, Video: Marangoni bursting: Evaporation-induced emulsification of a two-component droplet, https://doi.org/10.1103/APS.DFD.2017.GFM.V0020.

[7] G. Durey, H. Kwon, Q. Magdelaine, M. Casiulis, J. Mazet, L. Keiser, H. Bense, P. Colinet, J. Bico, and É. Reyssat, Marangoni bursting: Evaporation-induced emulsification of a two-component droplet, Phys. Rev. Fluids 3, 100501 (2018). 